\title{
Multiple coherent states semiclassical initial value representation spectra calculations of lateral interactions for $\mathrm{CO}$ on $\mathrm{Cu}(100)$
}

\author{
Michele Ceotto, ${ }^{1, a)}$ David Dell'Angelo, ${ }^{1, b)}$ and Gian Franco Tantardini ${ }^{1,2}$ \\ ${ }^{1}$ Dipartimento di Chimica Fisica ed Elettrochimica, Università degli Studi di Milano, \\ via Golgi 19, 20133 Milano, Italy \\ ${ }^{2}$ Istituto CNR di Scienze e Tecnologie Molecolari, via Golgi 19, 20133 Milano, Italy
}

(Received 4 May 2010; accepted 18 June 2010; published online 3 August 2010)

\begin{abstract}
Lateral interactions between carbon monoxide molecules adsorbed on a copper $\mathrm{Cu}(100)$ surface are investigated via semiclassical initial value representation (SC-IVR) molecular dynamics. A previous analytical potential is extended to include long-range dipole interactions between coadsorbed molecules and preliminary classical simulations were performed to tune the potential parameters. Then, the spectra for several coadsorbed molecules are calculated using the multiple coherent states approximation of the time-averaging representation of the SC-IVR propagator. Results show strong resonances between coadsorbed molecules as observed by past experiments. Resonances turn into dephasing when isotopical substitutions are performed. () 2010 American Institute of Physics.

[doi:10.1063/1.3462242]
\end{abstract}

\section{INTRODUCTION}

A number of methods ${ }^{1-4}$ that perform exact quantum diagonalization for eigenvalue and eigenfunction calculations have been developed in the past years. These methods have been implemented on a common base given by the discrete variable representation (DVR) and employed mostly for rotovibrational spectra of triatomic molecules. Implementations involved, for example, the implicitly restarted Lanczos method and distributed-memory parallelization, ${ }^{2}$ direct product of different basis ${ }^{1}$ (Wigner functions, Gauss-Legendre, Gauss-Hermite, Gauss-Lagueree, Gauss-Jacobi, etc.) or one-dimensional basis set optimization as in the case of potential optimized DVR. ${ }^{3}$ However, these are mostly confined to small molecules (especially triatomic) and for zero or small values of the total angular momentum. For large molecules, vibrational analysis has been often limited to harmonic normal mode pictures or to Monte Carlo ground state simulations. ${ }^{5}$ For these reasons, approximate methods have been developed. ${ }^{6-8}$ Recently, an implementation of the original time-averaging filtering of the semiclassical initial value representation method (SC-IVR) has been suggested, ${ }^{9}$ showing that accurate results for power spectra calculations can be achieved at a very low computational cost. These are comparable with those of the Monte Carlo phase space integration employing thousands of trajectories as will also be showed below. The multiple coherent states SC-IVR (MCSC-IVR) is a strategy for employing a basis set of a small number of coherent states, which resembles a linear combination of the eigenfunctions of the system. In this way, a multiple vibrational component signal is successfully reproduced which results in a well-defined spectrum. In this paper, MC-SC-IVR will be applied for studying the lateral interac-

\footnotetext{
${ }^{a)}$ Electronic mail: michele.ceotto@unimi.it.

${ }^{b}$ Present address: Equipe SIMPA, Institut de Physique de Rennes, CNRS UMR 6251, Université de Rennes I, 35042 Rennes, France.
}

tions between carbon monoxide molecules chemisorbed on copper $\mathrm{Cu}(100)$, after carefully developing a suitable potential energy analytical form. The reason for choosing this system is manifold: Carbon monoxide is one of the most extensively studied adsorbates and lateral interactions still represent a challenge for SC-IVR methods. To properly describe this kind of long-range interactions, one needs high resolution spectra and to be able to deal with floppy modes. Therefore, $\mathrm{CO}$ interaction with surfaces has constituted the model system for molecular chemisorption for many years: It is the reactant for some important catalytic processes, for example, the Fischer-Tropsch synthesis. Furthermore, it is ubiquitous, binding spontaneously to many surfaces. ${ }^{10}$

In a pioneering work about $\mathrm{CO}$ adsorption on metals, Hammaker et al. ${ }^{11}$ found that the major contribution to intermolecular interactions is of dipole type. They used an isotopic mixture of $\mathrm{CO}$ and looked at the intensity ratio and frequency changes as a function of coverage. Later, negligible adsorption was found to be present at room temperature whereas at lower temperatures, reversible adsorption was observed. ${ }^{12}$ Half monolayer is the maximum coverage due to the repulsive nature of the interadsorbate interaction. ${ }^{13}$ The infrared spectra showed ${ }^{14}$ a sharp and single very intense peak (for the internal $\mathrm{CO}$ stretch) which shifted from $2065 \mathrm{~cm}^{-1}$ at low coverage to $2101 \mathrm{~cm}^{-1}$ with increasing coverage and the shift was attributed to the strong dipoledipole coupling in the overlayer. ${ }^{14}$ A similar shift of about $40 \mathrm{~cm}^{-1}$ was found ${ }^{15}$ later, providing conclusive evidence that this is attributable to dipole coupling. Even a bigger shift $\left(77 \mathrm{~cm}^{-1}\right)$ was found for $\mathrm{CO}$ on $\mathrm{Ru}(0001) .{ }^{16}$ Thus, one comes to the conclusion that including lateral interactions is necessary for a realistic description of $\mathrm{CO}$ molecules adsorbed on metals surfaces. In this paper these interactions will be included into the potential analytic form, and we will also investigate their role in the observed frequency shift.

A comprehensive study of lateral interactions for $\mathrm{CO}$ on 
metals was performed by Persson and co-workers. ${ }^{17-20}$ They reproduced such frequency shift by creating a model based on a linear combination of atomic orbitals and second order perturbation theory. ${ }^{18}$ Later, they successfully used the coherent potential approximation employing only dipole interactions between adsorbed $\mathrm{CO} .{ }^{17}$ In this way, they showed that lateral interactions are crucial for a correct understanding of adsorbates monolayers in general and for $\mathrm{CO}$ on $\mathrm{Cu}(100)$ in particular. ${ }^{20}$ A collective mode behavior of vibrationally excited $\mathrm{CO}$ molecules sets up in the $c(2 \times 2)$ structure on $\mathrm{Cu}(100)$ (Ref. 21) and this induces both the frequency shift and the vibrational damping. ${ }^{22}$ The coupling is of dynamical nature and it has marked effects on the dephasing, such as on the temperature dependence of the linewidth and the line positions, as mentioned above. ${ }^{23}$ These lateral interactions promote energy delocalization processes which are competitive with vibrorotational relaxation ones. ${ }^{24}$ At the core of this behavior, there are dynamic dipole contributions, which are about twice the ones in the gas phase. Once the molecule is adsorbed, the $\mathrm{C}-\mathrm{O}$ bond stretching causes the $2 \pi^{*}$ level to lower at the Fermi level and to acquire a charge flow from the metal. ${ }^{17,25}$ This bond is weakened due to the increased population of the antibonding $\mathrm{CO} 2 \pi^{*}$ in the backdonation. This is the well known "Blyholder model, ${ }^{, 26}$ which has been set into question since a hybridization of the whole $\pi$-electronic structure must be considered. For a complete description, the reader could refer to Ref. 27, where a full hybridization of the initial $\mathrm{CO} \sigma$-molecular orbitals is observed. Actually, lateral interactions are dominant for adsorbated $\mathrm{CO}$ molecules on several metals, such as $\mathrm{Co}, \mathrm{Ni}, \mathrm{Cu}$, and $\mathrm{Fe}^{28}$ In summary, all experimental data can be consistently understood by employing a model where the $\mathrm{C}-\mathrm{O}$ stretch vibrations are coupled laterally via the long-range dipole interaction.

While most of computational studies have been focused on the single $\mathrm{CO}$ adsorbed molecule, very few simulations ${ }^{29-31}$ investigated intermolecular couplings. Bowman and co-workers ${ }^{30}$ showed that dipole coupling between two $\mathrm{CO}$ molecules results in an increase of the frequency of the $\mathrm{C}-\mathrm{O}$ stretch vibration and in a decrease of the width of the $\mathrm{C}-\mathrm{O}$ stretch spectral line. The dipole interaction drives the $\mathrm{CO}$ molecules further apart from their equilibrium positions, which is directly on top of the corresponding $\mathrm{Cu}$ atoms. However, such a repulsive shifting is small compared to the $\mathrm{CO}-\mathrm{Cu}$ binding energy ${ }^{30}$ as shown for four $\mathrm{CO}$ adsorbates. ${ }^{31}$ Nevertheless, their $5.5 \mathrm{~cm}^{-1}$ frequency shift is not realistic and they used a $\mathrm{CO}-\mathrm{CO}$ van der Waals interaction adapted from a multipole expansion describing $\mathrm{CO}$ molecules on $\mathrm{NaCl}(100) .{ }^{32}$ On the other side, first-principles calculations have been confined to a single $\mathrm{CO}$ adsorbed molecule. ${ }^{33-36}$ Thus, one has to rely on semiempirical potential analytical forms in the case of lateral interaction studies.

The aim of this work is to develop and test via classical and semiclassical simulations a potential energy surface able to describe energy delocalization and dephasing collective behaviors present in $\mathrm{CO}$ adsorbates on $\mathrm{Cu}(100)$. Quantum features of this system, such as vibrational energy levels, will be calculated at a semiclassical level of approximation. We believe that a potential which reproduces experimental infor- mation when used in quantum simulations is a solid starting point for future applications. Electron-hole (e-h) energy dissipation will not be included in our classical and semiclassical simulations. Indeed the e-h energy channel is significant and responsible for the vibrational line width since internal stretch and frustrated rotations are coupled to e-h pairs. ${ }^{37,38}$ Especially in scattering processes and sticking probability calculations, e-h pairs may be responsible for significant incident translational energies dissipation. ${ }^{29}$

In the next section the potential energy surface for $\mathrm{CO}$ adsorption layer on $\mathrm{Cu}(100)$ is revisited and in Sec. III classical simulation results are presented. In Sec. IV the semiclassical theory is recalled for spectra calculations and in Sec. V applied to the calculations of lateral interactions effects. Finally conclusions are drawn in Sec. VI.

\section{POTENTIAL ENERGY SURFACE}

The issue of the geometrical arrangement for $\mathrm{CO}$ adsorbed on $\mathrm{Cu}(100)$ has been controversial for some time. First, vibrational spectroscopy of $\mathrm{CO}$ adsorbed in the $c(2 \times 2)$ structure on $\mathrm{Cu}(100)$ and $\mathrm{Ni}(100)$ (Ref. 39) showed that the molecule adsorbs in a terminal configuration, i.e., on top of a metal surface atom with the carbon bonded to the metal. When a CO molecule adsorbs on top of a copper atom, besides the internal $\mathrm{C}-\mathrm{O}$ stretch mode (I-mode, $\nu_{1}$ ), there are five external ones: the twofold degenerate frustrated rotations (R-mode, $\nu_{2}$ ), the twofold degenerate frustrated translations (T-mode, $\nu_{4}$ ), and the $\mathrm{C}-\mathrm{Cu}$ stretch (S-mode, $\nu_{3}$ ) with respect to the surface. For what regard symmetry, the free $\mathrm{CO}$ molecule passes from a $C_{\infty_{v}}$ group to a $C_{4 v}$ group. Adsorption onto a high symmetry on-top site such as $C_{4 v}$ results in degeneracies. However the orientation of the molecule was an open dispute for years. The ultraviolet photoelectron spectroscopy (UPS) results by Plummer and co-workers ${ }^{40}$ indicated that the molecule stands with its axis perpendicular to the surface with the carbon end down, and with a possible deviation from this orientation being of the order of $10^{\circ}-15^{\circ}$. On the other hand, dynamical low-energy electron diffraction (LEED) methods ${ }^{41}$ took the side of a skewed molecule, also with the carbon end down on a topatom site but inclined at an angle of $34^{\circ}$ with respect to the surface's normal. A later calculation ${ }^{42}$ attempted to place an end to the controversy by showing that the contraction of a molecular bond is possible upon chemisorption and hence that tilting of the $\mathrm{CO}$ molecule is not necessary. One year later, in LEED experiments by Passler and co-workers, ${ }^{43}$ particular attention was paid to the preparation of the $\mathrm{CO}$ structure and to the minimization of the incident electron current during collection of LEED intensity data. All structural models tested had the $\mathrm{CO}$ molecule perpendicular to the surface, with the carbon end down. Finally, x-ray absorption and photoelectron diffraction experiments ${ }^{44}$ concluded the molecule orientation to be perpendicular to the surface. A summary of experimental results is reported in Table I. Infrared experiments were performed on this system for a long time because of the strong signal of the intramolecular CO stretch. However, external stretch and frustrated dipole forbidden rotations and translations were revealed only by far-infrared 
TABLE I. Available experimental data for $\mathrm{CO} / \mathrm{Cu}(100)$ system: $\mathrm{C}-\mathrm{O}$ bond length $\left(d_{\mathrm{C}-\mathrm{O}}\right), \mathrm{C}-\mathrm{Cu}$ bond length $\left(d_{\mathrm{C}-\mathrm{Cu}}\right)$, equilibrium lattice constant for bulk $\mathrm{Cu}$ (a), dipole moment for the CO molecule $\left(\mu_{0}\right)$, and $\mu^{\prime}$ is its first derivative respect to the CO distance. The $\nu_{1}, \nu_{2}, \nu_{3}, \nu_{4}$ frequency values (wavenumbers) correspond to the I-mode (internal symmetric stretch), R-mode (frustrated rotations), S-mode (external symmetric stretch), and T-mode (frustrated translations), respectively.

\begin{tabular}{|c|c|c|c|c|}
\hline \multicolumn{5}{|c|}{ Expt. data } \\
\hline$d_{\mathrm{C}-\mathrm{O}}(\AA)$ & $1.1^{\mathrm{a}}$ & $1.15^{\mathrm{b}}$ & $1.15^{\mathrm{c}, \mathrm{d}}$ & $1.18^{\mathrm{e}}$ \\
\hline$d_{\mathrm{Cu}-\mathrm{C}}(\AA)$ & & $1.92^{\mathrm{c}, \mathrm{f}}$ & $1.9^{\mathrm{c}, \mathrm{d}}$ & $1.92^{\mathrm{e}}$ \\
\hline$a(\AA)$ & $3.62^{\mathrm{g}}$ & & & \\
\hline$\mu_{0}$ (a.u.) & $0.112^{\mathrm{h}}$ & $0.18^{\mathrm{i}, \mathrm{j}}$ & $0.27^{\mathrm{k}, \mathrm{j}}$ & $0.27^{1}$ \\
\hline$\mu^{\prime}$ (a.u.) & & & $-2.25^{\mathrm{m}}$ & $-2.25^{1}$ \\
\hline
\end{tabular}

\begin{tabular}{|c|c|c|c|c|}
\hline \multirow[b]{2}{*}{ Reference/year } & \multicolumn{3}{|c|}{ Expt. frequency } & \multirow[b]{2}{*}{$\begin{array}{c}\nu_{4} \\
\text { (translation) }\end{array}$} \\
\hline & $\begin{array}{c}\nu_{1} \\
\text { (internal stretching) }\end{array}$ & $\begin{array}{c}\nu_{2} \\
\text { (rotation) }\end{array}$ & $\begin{array}{c}\nu_{3} \\
\text { (external stretching) }\end{array}$ & \\
\hline 45 and $46 / 1990$ & 2084.6 & 285 & 345 & \\
\hline 47 and $48 / 1993$ & 2086 & 285 & 345 & 32 \\
\hline $49 / 1994$ & 2078 & 287 & 344 & \\
\hline $50 / 2006$ & 2079 & & & \\
\hline In this work & 2081 & 295 & 343 & 19 \\
\hline
\end{tabular}

${ }^{\mathrm{a}}$ Reference 12 .

${ }^{\mathrm{b}}$ This value is for $\mathrm{CO}$ adsorbed on a $\mathrm{Ni}(001)$ surface; Ref. 43.

${ }^{\mathrm{c}} \pm 0.1 \AA$ A uncertainty.

${ }^{\mathrm{d}}$ Reference 51 .

${ }^{e}$ References 45 and 46 .

${ }^{\mathrm{f}}$ Reference 44.

${ }^{g}$ Reference 52; $\pm 0.05 \AA$ A uncertainty.

(FIR) measurements using synchrotron radiation. ${ }^{46}$ The final geometry, including the solid fcc solid copper is shown in Fig. 1.

The analytical potential developed by Tully and co-workers ${ }^{29,37,56}$ is at the basis of almost all theoretical and molecular dynamics simulations of the $\mathrm{CO}$ on the $\mathrm{Cu}(100)$ system. Several groups employed this potential for vibrational analysis ${ }^{29-31,55,57-61}$ and explored how modes are coupled $^{57}$ and the eventual discrepancy between experiments and theory. ${ }^{59}$ In a very recent work by Marquardt et al. ${ }^{34}$ a new global analytical representation of the potential energy surface for this process is derived to better fit electronic structure data. Anharmonic mode frequencies are in good agreement with experimental data.

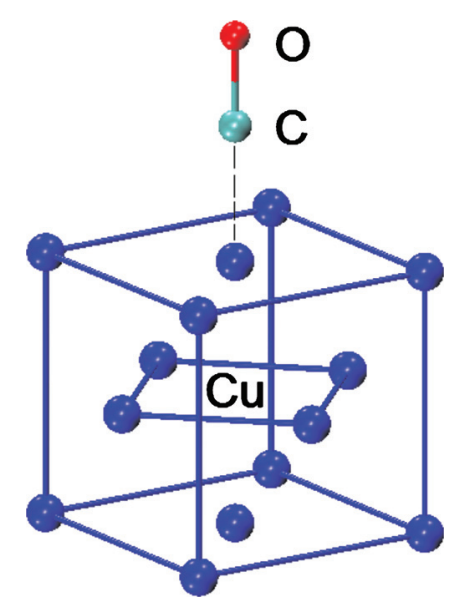

FIG. 1. Geometrical arrangement of the carbon monoxide adsorption on a $\mathrm{Cu}(100)$ surface.

\author{
${ }^{\mathrm{h}}$ Gas phase data; Ref. 53 . \\ ${ }^{\mathrm{i}}$ From IR intensity and EELS intensity experiments. \\ ${ }^{\mathrm{j}}$ Reference 19. \\ ${ }^{\mathrm{k}}$ From IR isotopic mixture and EELS normal mode dispersion experiments. \\ ${ }^{\mathrm{I}}$ From $a b$ initio multi-configurational self-consistent field (MCSCF) calcula- \\ tions; Ref. 54. \\ ${ }^{\mathrm{m}}$ Reference 55 .
}

In this paper, Tully's analytical potential will be reexamined and implemented to properly describe lateral interactions. The potential form is

$$
V\left(\mathbf{r}_{\mathrm{C}}, \mathbf{r}_{\mathrm{O}}\right)=\sum_{i=1}^{N_{\text {copper }}} V_{i}\left(\mathbf{r}_{\mathrm{C}}, \mathbf{r}_{\mathrm{O}}, \mathbf{r}_{i}\right)+V_{\mathrm{CO}}\left(\left|\mathbf{r}_{\mathrm{C}}-\mathbf{r}_{\mathrm{O}}\right|\right),
$$

where $\mathbf{r}_{\mathrm{C}}$ and $\mathbf{r}_{\mathrm{O}}$ are the carbon and the oxygen position vectors, respectively, and $\mathbf{r}_{i}$ are those of the $i$ th coordinate of the copper atom. The intramolecular term $V_{\mathrm{CO}}$ is a standard Morse potential

$$
\begin{aligned}
V_{\mathrm{CO}}\left(\left|\mathbf{r}_{\mathrm{C}}-\mathbf{r}_{\mathrm{O}}\right|\right)= & F\left\{\exp \left(-2 \gamma\left(\left|\mathbf{r}_{\mathrm{C}}-\mathbf{r}_{\mathrm{O}}\right|-r_{\mathrm{CO}}\right)\right)\right. \\
& \left.-2 \exp \left(-\gamma\left(\left|\mathbf{r}_{\mathrm{C}}-\mathbf{r}_{\mathrm{O}}\right|-r_{\mathrm{CO}}\right)\right)\right\},
\end{aligned}
$$

where $r_{\mathrm{CO}}$ is the equilibrium distance, and the interaction of $\mathrm{CO}$ with each copper atom is described by the following modified Morse $\mathrm{C}-\mathrm{Cu}$ interaction potential

$$
\begin{aligned}
V_{i}\left(\mathbf{r}_{\mathrm{C}}, \mathbf{r}_{\mathrm{O}}, \mathbf{r}_{i}\right)= & A \exp \left(-\alpha\left|\mathbf{r}_{i}-\mathbf{r}_{\mathrm{O}}\right|\right) \\
& +B \exp \left(-2 \beta\left(\left|\mathbf{r}_{i}-\mathbf{r}_{\mathrm{C}}\right|-r_{\mathrm{eq}}\right)\right) \\
& -2 B \cos \phi^{2} \exp \left(-\beta\left(\left|\mathbf{r}_{i}-\mathbf{r}_{\mathrm{C}}\right|-r_{\mathrm{eq}}\right)\right),
\end{aligned}
$$

where $r_{\mathrm{eq}}$ is a given equilibrium distance and

$$
\cos ^{2} \phi=\frac{\left(\mathbf{r}_{i}-\mathbf{r}_{\mathrm{C}}\right) \cdot\left(\mathbf{r}_{\mathrm{C}}-\mathbf{r}_{\mathrm{O}}\right)}{\left|\mathbf{r}_{i}-\mathbf{r}_{\mathrm{C}}\right|\left|\mathbf{r}_{\mathrm{C}}-\mathbf{r}_{\mathrm{O}}\right|}
$$

The first term in Eq. (3) describes the oxygen-copper repulsion. The remainder of the terms in Eq. (3) represents the carbon-copper interaction. The molecule orientation is taken into account by the angle $\phi$ between the $\mathrm{C}-\mathrm{O}$ and the $\mathrm{Cu}-\mathrm{C}$ bonds. The metal is represented by three layers of 
TABLE II. Parameter values used in Eqs. (2) and (3).

\begin{tabular}{llcc}
\hline \hline Parameter & Value & Parameter & Value \\
\hline$r_{\mathrm{CO}}(\AA)$ & 1.25 & $\mathrm{~A}\left(\mathrm{~kJ} \times \mathrm{mol}^{-1}\right)$ & 84300.0 \\
$r_{e}(\AA)$ & 1.9 & $\mathrm{~B}\left(\mathrm{~kJ} \times \mathrm{mol}^{-1}\right)$ & $50.93^{\mathrm{a}}$ \\
$\alpha\left(\AA^{-1}\right)$ & 3.36 & $\mathrm{~F}\left(\mathrm{~kJ} \times \mathrm{mol}^{-1}\right)$ & 1070.0 \\
$\beta\left(\AA^{-1}\right)$ & 3.5 & $\gamma\left(\AA^{-1}\right)$ & 2.16 \\
\hline
\end{tabular}

${ }^{\mathrm{a}}$ Experimental value of $52.71 \mathrm{~kJ} / \mathrm{mol}$ (Refs. 12 and 62).

$36(6 \times 6)$ copper atoms arranged according to a fcc lattice structure. By inspecting the several theoretical papers that make use of this potential form, one can find some inconsistencies in the normal mode vibrational frequencies' values. ${ }^{29-31,55,57-61}$ The set of parameters used in this work for Eqs. (2) and (3) are reported in Table II to be matched with the experimental ones in Table I. The effect of each parameter in Eqs. (1)-(3) on the global potential and on the normal modes frequencies has been studied. Given the experimental lattice constant of $3.62 \AA,{ }^{29,37,53}$ we found that to better describe the experimental values of the frequencies, one has to lower by $-0.4761 \AA$ the zeta coordinate of the adsorption site copper atom and by $-0.2381 \AA$ that one of the four nearest-neighbors. In this way we have obtained the frequency values reported in the last row of Table I. However, density functional theory (DFT) calculations suggest an opposite deviation for the zeta coordinate. ${ }^{63}$ The classical desorption energy reported in Table II is also very close to the experimental value if the zero point energy is subtracted. The lattice dimension was also tested with the frequency values and a $6 \times 6$ surface layer was confirmed as fully converged. This is shown in Fig. 2.

When considering a monolayer of $\mathrm{CO}$ molecules, one should not only sum Eq. (1) for each molecule but also an extra pairwise potential term to describe eventual interactions between several $\mathrm{CO}$ molecules. The collective behavior of this monolayer has been studied by using the screened image potential ${ }^{17,64}$ and these findings clearly suggest that the molecules interact mainly through their dipole fields and the phonon mediated coupling between two $\mathrm{CO}$ molecules can be disregarded. ${ }^{17-20,22}$ Clearly, for an array of closely

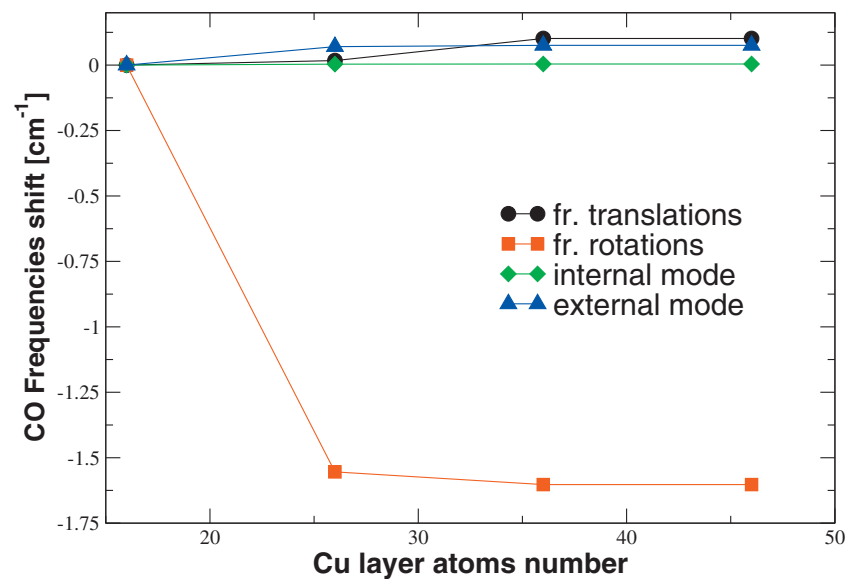

FIG. 2. Test for a suitable substrate dimension: a six by six layer of copper atoms is converged even with respect to the most sensitive frustrated rotations frequency. spaced molecules, e.g., a layer of molecules adsorbed on a surface, the lateral coupling between the high-frequency vibrational modes will become of fundamental importance. The difference in the singleton frequencies, from the one at $\mathrm{CO}$ coverage $0.33 \mathrm{ML}$ (monolayer) versus the one at $0.0025 \mathrm{ML}$ (both measured at $30 \mathrm{~K}$ ) reflected a chemical shift of the $\mathrm{C}-\mathrm{O}$ stretching mode induced by surrounding $\mathrm{CO}$ molecules. ${ }^{23}$ Thus, a suitable estimate of the dynamic dipole moment $\mu_{D}$ at the adsorption geometry is, to a very good approximation, equal to the derivative of the dipole moment operator respect to the internal mode stretch averaged over the ground state electronic wave function. ${ }^{54}$ For these reasons, the dipole interactions between each pair of molecule were chosen to be

$$
V_{12}(\mathbf{r})=-\mu_{1} \mu_{2} \cos \theta_{12} / r^{3},
$$

where $\cos \theta_{12}$ is the angle between the bond directions of the two molecules, $r$ is the distance between the two molecules, and $\mu_{1}$ and $\mu_{2}$ are the dipole moment of each molecule. In order to properly describe the dynamical dipole interactions, the dipole moment is expanded at its first order,

$$
\mu\left(\mathbf{r}_{\mathrm{CO}}\right)=\mu_{0}+\frac{d \mu\left(\mathbf{r}_{\mathrm{CO}}\right)}{d \mathbf{r}_{\mathrm{CO}}}\left(\mathbf{r}_{\mathrm{CO}}-\mathbf{r}_{\mathrm{CO}, \mathrm{eq}}\right) .
$$

In this work the permanent dipole $\mu_{0}$ and the transition dipole $d \mu\left(\mathbf{r}_{\mathrm{CO}}\right) / d \mathbf{r}_{\mathrm{CO}}=\mu^{\prime}$ moments' values are that ones reported in the last raw of Table I.

\section{CLASSICAL SIMULATIONS}

Classical molecular dynamics simulations were performed on a single adsorbed molecule. Several algorithms for time propagation have been tested and the one used ${ }^{65}$ both for the classical and semiclassical simulations reported has proven to preserve energy six orders of magnitude better than the velocity Verlet algorithm. In Table I, the normal mode frequencies from the Hessian diagonalization are reported and matched with the experimental ones as has been done in previous discussions about this potential. ${ }^{37,55,59-61}$ However more properly one should perform this comparison only after taking into account eventual anharmonic effects. Velocity autocorrelation functions were employed via the Fourier transform for calculating the normal mode spectra reported in Fig. 3 in order to unmask and quantify anharmonic effects. ${ }^{30,31,57,58}$ Classical molecular dynamics on a harmonic approximation of the potential model in Eq. (1) and on the actual potential were performed. The results reported in Fig. 3 show that anharmonicity lowers both the internal and the external stretching frequency. Instead it has an opposite effect on the rotational ones, which are stiffer. ${ }^{57}$ However, the amount of these deviations and of the coupling between modes is very much contained, such that the Hessian diagonalization is still valid for comparison with experimental results. Then, velocity channeling simulations have been performed in order to understand the nature of the intermode coupling. Energy transfer between modes is a key ingredient during the adsorption process because it induces molecular trapping as well as desorption. No significant coupling has been found between the internal stretching mode 


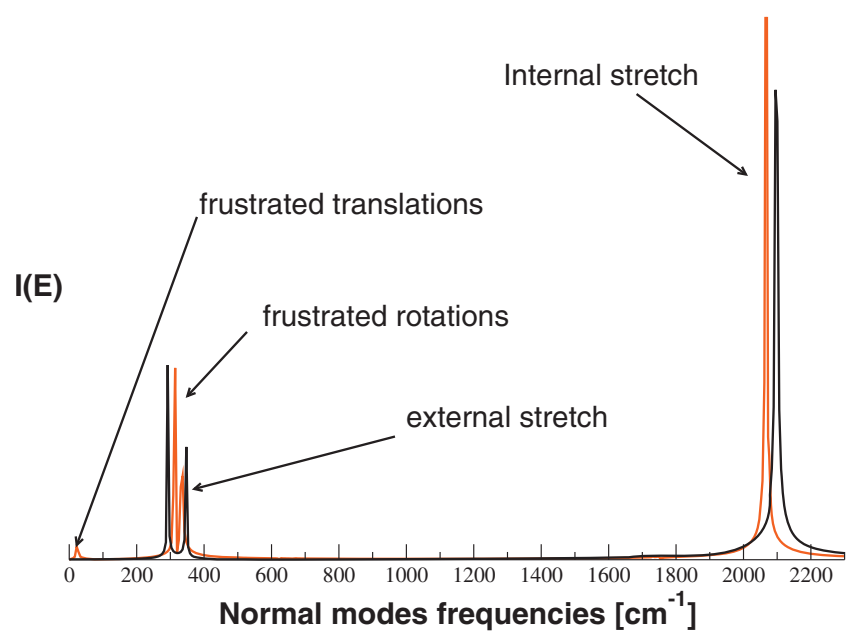

FIG. 3. Harmonic (black) and exact (red) normal mode spectra of a single molecule adsorbed on $\mathrm{Cu}(100)$.

and the external ones, suggesting that eventual internal excited states will survive. Instead coupling has been found between the external ones, in particular, between the rotations and translations. Finally, classical simulations have been performed for different isotopic species of $\mathrm{C}$ and $\mathrm{O}$ to see how this potential can explain why many experimental teams can detect changes in spectrum by using different isotopic mixtures. As one may expect, frequency shifts were detected only for the internal stretching mode, while leaving the frequency peaks of the external modes unchanged. The isotope ${ }^{12} \mathrm{C}^{18} \mathrm{O}$ lowers the I-mode by roughly $15 \mathrm{~cm}^{-1}$, the ${ }^{14} \mathrm{C}^{16} \mathrm{O}$ one by $40 \mathrm{~cm}^{-1}$, and the ${ }^{14} \mathrm{C}^{18} \mathrm{O}$ one by $60 \mathrm{~cm}^{-1}$.

\section{MULTIPLE COHERENT STATES SEMICLASSICAL INITIAL VALUE REPRESENTATION SIMULATIONS}

In this work, spectra will be calculated using the multiple coherent state time-averaging semiclassical initial value representation approximation (MC-SC-IVR), recently developed in our group. ${ }^{9}$ In the SC-IVR method, ${ }^{65-73}$ the propagator in $F$ dimensions is approximated by the phase space integral,

$$
\begin{aligned}
e^{-i \hat{H} t / \hbar}= & \frac{1}{(2 \pi \hbar)^{F}} \int d \mathbf{p}(0) \int d \mathbf{q}(0) C_{t}(\mathbf{p}(0), \mathbf{q}(0)) \\
& \times e^{i S_{t}(\mathbf{p}(0), \mathbf{q}(0)) / \hbar}|\mathbf{p}(t), \mathbf{q}(t)\rangle\langle\mathbf{p}(0), \mathbf{q}(0)|,
\end{aligned}
$$

where $(\mathbf{p}(t), \mathbf{q}(t))$ are the set of classically evolved phase space coordinates, $S_{t}$ is the classical action, and $C_{t}$ is a preexponential factor. In the Heller-Herman-Kluk-Kay ${ }^{74,75}$ version of the SC-IVR, the prefactor is

$$
\begin{aligned}
& C_{t}(\mathbf{p}(0), \mathbf{q}(0)) \\
& \quad=\sqrt{\frac{1}{2}\left|\frac{\partial \mathbf{q}(t)}{\partial \mathbf{q}(0)}+\frac{\partial \mathbf{p}(t)}{\partial \mathbf{p}(0)}-i \hbar \gamma \frac{\partial \mathbf{q}(t)}{\partial \mathbf{p}(0)}+\frac{i}{\gamma \hbar} \frac{\partial \mathbf{p}(t)}{\partial \mathbf{q}(0)}\right|}
\end{aligned}
$$

and the basis set is a product of $F$ one-dimensional Gaussians $\langle\mathbf{q} \mid \mathbf{p}(t), \mathbf{q}(t)\rangle=\Pi_{i}\left(\gamma_{i} / \pi\right)^{F / 4} \exp \left[-\gamma_{i} \cdot\left(q_{i}-q_{i}(t)\right) / 2\right.$ $\left.+i p_{i}(t) \cdot\left(q_{i}-q_{i}(t)\right) / \hbar\right]$ of fixed width $\gamma_{i}$. For bound systems, the widths are usually chosen to match the widths of the harmonic oscillator approximation to the wave function at the global minimum and no significant dependency has been found under width variation. ${ }^{68}$ By using a $2 F \times 2 F$ symplectic (monodromy) matrix $\mathbf{M}(t) \equiv \partial\left(\left(\mathbf{p}_{t}, \mathbf{q}_{t}\right) / \partial\left(\mathbf{p}_{0}, \mathbf{q}_{0}\right)\right)$, one can calculate the prefactor of Eq. (8) from blocks of $F \times F$ size and we monitored the accuracy of the classical approximate propagation by containing the deviation of its determinant from unity to $10^{-5}$.

A useful way for molecular dynamics to evaluate a spectral density is as a Fourier transform of an autocorrelation function with respect to an arbitrary reference state $|\chi\rangle$ $=\left|\mathbf{p}_{N}, \mathbf{q}_{N}\right\rangle{ }^{76}$ In the specific case of our spectra, the reference state is chosen as a mix of vibrational states operated by the dipole moment,

$$
I(E)=\frac{\operatorname{Re}}{\pi \hbar} \int_{0}^{+\infty} e^{i E t / \hbar}\left\langle\chi \hat{\mu}\left|e^{-i \hat{H} t / \hbar}\right| \hat{\mu} \chi\right\rangle d t,
$$

where $\hat{\mu}$ is the dipole operator and $E$ is the energy of the system. If $\hat{\mu}$ is set to identity, one would calculate the power spectrum and the values of $E$ at peak positions will indicate the vibrational energy levels, including $E_{g}$, the ground state energy level. Instead, if $\hat{\mu}$ is set to the dipole moment operator, the energy value in the Fourier transform is shifted with respect to the ground state energy, i.e., $E \rightarrow E+E_{g}$ and $E$ is the spectrum variable, $I(E)$. We did a preliminary semiclassical power spectrum computation to evaluate $E_{g_{g}}$ and then the spectrum with the dipole operator was shifted. ${ }^{97}$ The values of $E_{g}$ were accurate enough to well reproduce the elastic peak at the origin of the spectrum on each simulation. Equation (9) can be approximated semiclassically by inserting Eq. (7) as the evolution operator. The phase space integral of Eq. (7) is usually computed using Monte Carlo methods. If the simulation time is long enough, the phase space average can be well approximated by a time-averaging integral. Using this idea, one obtains the time-averaging ${ }^{78}$ (TA) (Ref. 6) SCIVR approximation for Eq. (9) is ${ }^{79}$

$$
\begin{aligned}
I(E)= & \frac{1}{(2 \pi \hbar)^{F}} \int d \mathbf{p}(0) \int d \mathbf{q}(0) \\
& \times \frac{\operatorname{Re}}{\pi \hbar T} \int_{0}^{T} d t_{1} \int_{t_{1}}^{T} d t_{2} C_{t_{2}}\left(\mathbf{p}\left(t_{1}\right), \mathbf{q}\left(t_{1}\right)\right) \\
& \times\left\langle\chi \hat{\mu} \mid \mathbf{p}\left(t_{2}\right), \mathbf{q}\left(t_{2}\right)\right\rangle e^{i\left(S_{t_{2}}(\mathbf{p}(0), \mathbf{q}(0))+E t_{2}\right) / \hbar} \\
& \times\left[\left\langle\chi \hat{\mu} \mid \mathbf{p}\left(t_{1}\right), \mathbf{q}\left(t_{1}\right)\right\rangle e^{i\left(S_{t_{1}}(\mathbf{p}(0), \mathbf{q}(0))+E t_{1}\right) / \hbar}\right]^{*},
\end{aligned}
$$

where $\left(\mathbf{p}\left(t_{1}\right), \mathbf{q}\left(t_{1}\right)\right)$ and $\left(\mathbf{p}\left(t_{2}\right), \mathbf{q}\left(t_{2}\right)\right)$ are variables that evolve from the same initial conditions but to different times, and $T$ is the total simulation time. In Eq. (10), the first time integral is the Fourier transform of Eq. (9) and the second time integration corresponds to the time-averaging filter. The double-time integration implies that for any given trajectory from time 0 to time $T$, all possible intervals from $t_{1}$ to $t_{2}$ are considered. The advantage of this approach is that the additional time integral can, in principle, replace the need for phase space averaging in the large-time limit of a single trajectory. In order to make Eq. (10) less computationally demanding, one can employ the separable approximation, ${ }^{6}$ where the prefactor of Eq. (10) is approximated as a phase, $C_{t_{2}}\left(\mathbf{p}\left(t_{1}\right), \mathbf{q}\left(t_{1}\right)\right)=\exp \left[i\left(\phi\left(t_{2}\right)-\phi\left(t_{1}\right)\right) / \hbar\right]$, and $\phi(t)$ 
$=$ phase $\left[C_{t}(\mathbf{p}(0), \mathbf{q}(0))\right]$. Using this approximation, Eq. (10) becomes

$$
\begin{aligned}
I(E)= & \frac{1}{(2 \pi \hbar)^{F}} \frac{1}{2 \pi \hbar T} \int d \mathbf{p}(0) \int d \mathbf{q}(0) \\
& \times \mid \int_{0}^{T} d t\langle\chi \hat{\mu} \mid \mathbf{p}(t), \mathbf{q}(t)\rangle \\
& \times\left. e^{i\left(S_{t}(\mathbf{p}(0), \mathbf{q}(0))+E t+\phi_{t}(\mathbf{p}(0), \mathbf{q}(0)) / \hbar\right)}\right|^{2}
\end{aligned}
$$

leading to a simplification of the double-time integration to a single time integral. Our past numerical tests ${ }^{7,9}$ showed that the results of carrying out this approximation are most of the time as accurate as for the double-time integral approach.

The Monte Carlo phase space integration of Eqs. (11) and (10) has been tested to convergence to about a thousand of trajectories per each degree of freedom. ${ }^{6}$ This number of trajectories is greatly reduced with respect to a semiclassical approach without the time-averaging filter, however not enough for first-principles approaches to semiclassical dynamics ${ }^{7,9}$ or for the complex system we are going to tackle in this paper. For these reasons, we have recently developed the MC-SC-IVR, ${ }^{9}$ a SC-IVR strategy that enhances as much as possible the overlap between the reference state $|\chi\rangle$, and the exact quantum eigenfunctions. Such a strategy exhibits high intensity peaks even for excited states and this is of relevance because we have found by numerical simulation that usually the more intense the peaks are, the higher the accuracy obtained for a fixed-time simulation. To reach such a goal, one can think to project the power spectrum into the phase space portrait, where the spectrum is represented by a set of multidimensional closed trajectories with energy shell equal to the corresponding spectral peak (see Fig. 1 in Ref. 9). Then, one can choose a set of "eigentrajectories" having the turning points roughly located at the values of potential energy equal to the peaks of the power spectrum. An exact location of these states with respect to the peak location is not necessary since the Gaussian spreading of each coherent states is generally wide enough to include the peak energy shell, as we tested numerically in Ref. 9. In the MC-SC-IVR the reference states $|\chi\rangle$ were chosen to be a combination of coherent states,

$$
|\chi\rangle=\sum_{i=1}^{N_{\text {states }}}\left|\mathbf{p}_{\mathrm{eq}}^{\mathbf{i}}, \mathbf{q}_{\mathrm{eq}}^{\mathbf{i}}\right\rangle,
$$

located at the equilibrium molecular position $\mathbf{q}_{\mathrm{eq}}$ and the harmonic approximation is employed to set these initial momenta, namely, $p_{\text {eq }}^{2} / 2 m=\hbar \omega(n+1 / 2)$. In this way MC-SCIVR includes quantum mechanical delocalization by using a set of coherent states placed in a nonlocal fashion, while their centers are kept fixed all along the simulation time. By inserting Eq. (12) into Eq. (10) the final expression for the multiple coherent states spectra calculations used in this paper is

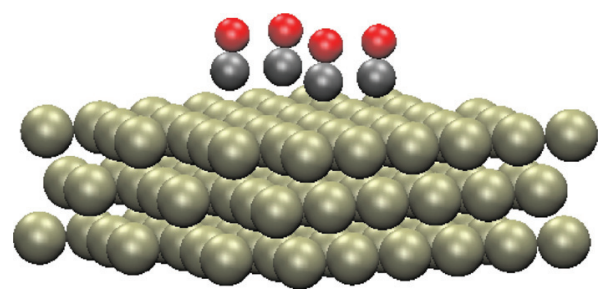

FIG. 4. Four $\mathrm{CO}$ molecule adsorption geometry on a $\mathrm{Cu}(100)$ surface. The solid one is made of three layers of six by six copper atoms and the $\mathrm{CO}$ molecules are placed in a half-monolayer fashion.

$$
\begin{aligned}
I(E)= & \frac{1}{(2 \pi \hbar)^{F}} \frac{\mathbf{R e}}{\pi \hbar T} \sum_{j=1}^{N_{\text {states }}} \int_{0}^{T} d t_{1} \int_{t_{1}}^{T} d t_{2} C_{t_{2}}\left(\mathbf{p}\left(t_{1}\right), \mathbf{q}\left(t_{1}\right)\right) \\
& \times \sum_{i=1}^{N_{\text {states }}}\left\langle\mathbf{p}_{\mathrm{eq}}^{\mathbf{i}}, \mathbf{q}_{\mathrm{eq}}^{\mathbf{i}} \hat{\mu} \mid \mathbf{p}^{\mathbf{j}}\left(t_{2}\right), \mathbf{q}^{\mathbf{j}}\left(t_{2}\right)\right\rangle e^{i\left(S_{t_{2}}(\mathbf{p}(0), \mathbf{q}(0))+E t_{2}\right) / \hbar} \\
& \times\left[\sum_{i=1}^{N_{\text {states }}}\left\langle\mathbf{p}_{\mathrm{eq}}^{\mathbf{i}}, \mathbf{q}_{\mathrm{eq}}^{\mathbf{i}} \hat{\mu} \mid \mathbf{p}^{\mathbf{j}}\left(t_{1}\right), \mathbf{q}^{\mathbf{j}}\left(t_{1}\right)\right\rangle e^{i\left(S_{t_{1}}(\mathbf{p}(0), \mathbf{q}(0))+E t_{1}\right) / \hbar}\right],
\end{aligned}
$$

where the phase space integral is now reduced to a sum over convenient coherent states centers. An analogous expression holds when the separable approximation is applied. When the single ground vibrational state is used as the reference state, one recovers the absorption cross-section at $0 \mathrm{~K}$. This is reported below for a 14000 trajectory simulation. Instead, when the multiple coherent state is used, physical information is given by the peak location and not from their relative intensities.

In this way, we obtained with few trajectories accurate results for $\mathrm{H}_{2} \mathrm{O}$ molecule on a model potential and for $\mathrm{CO}_{2}$ molecule using an on-the-fly approach. In particular, the spectra were very satisfying for the potential of Eq. (1) when dimensionality was reduced to the stretching motions ${ }^{9}$ since precision was enhanced when multiple coherent states were used. This approach is really advantageous since it has been proven that the number of trajectories can be substantially reduced. ${ }^{9}$

\section{LATERAL INTERACTIONS}

The main objective of this paper is to study the effect of the lateral interactions between coadsorbed $\mathrm{CO}$ molecules on a $\mathrm{Cu}(100)$ surface. In order to pursue such a goal, one needs to calculate the spectra of $\mathrm{CO}$ molecules arranged in a way to mimic the experimental coverage. The maximum coverage is reached when $\mathrm{CO}$ molecules represent $0.5 \mathrm{ML}$, i.e., in a $c(2 \times 2)$ geometry on $\mathrm{Cu}(100)$ surface. The long-range dipole interactions between coadsorbed molecules are weak and their effect on the spectra can be detected only by using quantum mechanical methods that can reach a resolution of a few wavenumbers. For these reasons the spectra of the system in Fig. 4 represents a challenging test for theoretical quantum methods. Previous quantum approaches using Eq. (9), such as the multi-configuration time-dependent Hartree (MCTDH) method reported in Ref. 60, were limited to a 


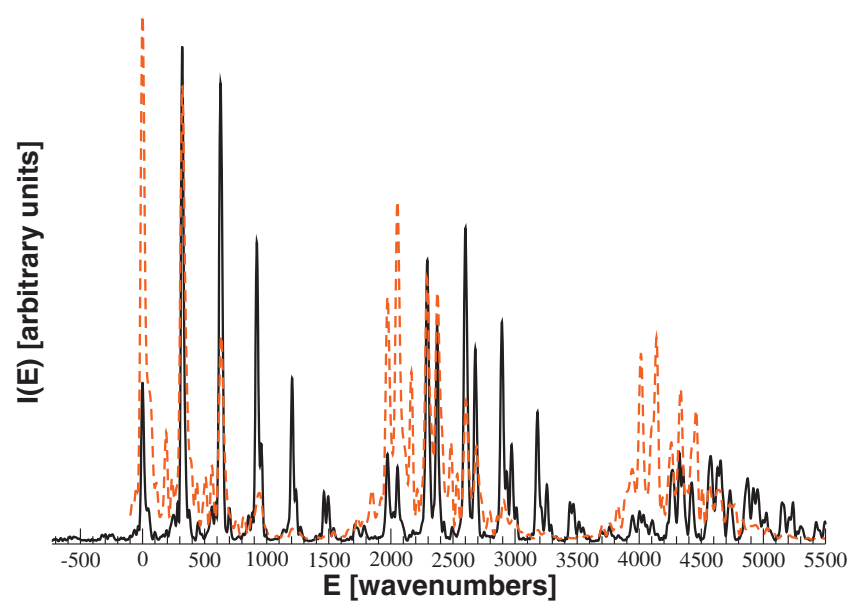

FIG. 5. Spectra for two CO molecules: continuous (black) line using MCTA-SC-IVR with 14 trajectories; dashed (red) line TA-SC-IVR with 14,000 trajectories.

single $\mathrm{CO}$ molecule. Instead other quantum calculations on several $\mathrm{CO}$ molecules on $\mathrm{Cu}(100)$ used a different coupling term. $^{30,31}$

At the beginning a system of only two $\mathrm{CO}$ molecules was studied. A set of classical simulations was performed in order to inspect the role of each mode in lateral interactions. These results clearly indicated that frustrated rotations and translations are minimally involved. Mostly the carbon atoms rotate around the axis perpendicular to the surface, while the oxygen rotation radii are very small. These motions do not alter significantly the dipole moment vector on each molecule. For these reasons, we have chosen to freeze these modes at their equilibrium geometry and allow the atoms to move only along the rotational axes, i.e., along the on-top direction to the surface. In this way the dimensionality was reduced to four in the case of a pair of $\mathrm{CO}$ molecules and to eight in the case of the system reported in Fig. 4. A closer inspection showed that lateral interactions are mostly induced by the internal stretching motion and only in minor part by the external stretching one. This is confirmed by a simple harmonic analysis which shows that external stretch, frustrated rotations, and translations have degenerate frequency values for different molecules, while the internal stretching frequencies are split. Experiments detected only a shift of the order of $40 \mathrm{~cm}^{-1} \cdot{ }^{11,14,15,21}$ In all semiclassical simulations reported here the time step used for solving Eqs. (10) and (11) was 0.024 fs and a typical trajectory length was 10000 steps. Such a long trajectory is needed in order to fully take advantage of the time-averaging filter and reach a spectra resolution of $1 \mathrm{~cm}^{-1}$. The resulting spectra for this system are reported in Fig. 5, where the MC-SC-IVR is compared with the SC-IVR and where both use the timeaveraging filter of course. The SC-IVR simulations employed 14000 trajectories: Taking into account that on average when using the time-averaging method one needs about 1000 trajectories per degree of freedom, ${ }^{6}$ these calculations are converged. In the $0-1500 \mathrm{~cm}^{-1}$ frequency range one can observe the external stretch vibrational energy levels. The two simulations give the same frequency peak location, even if for 14000 trajectories one is noisier and the peak magnitude smaller than with the MC-SC-IVR trajectories. In the $1900-3500 \mathrm{~cm}^{-1}$ frequency range an internal stretch quantum excitation is added to the external ones. In this case the splitting induced by the dipole coupling is clear and it is about $80 \mathrm{~cm}^{-1}$, in agreement with the experimental shift of $40 \mathrm{~cm}^{-1}$ for the internal stretch frequency. In this range as well, MC-SC-IVR shows a less noisy spectrum. The situation is the opposite in the $3500-5500 \mathrm{~cm}^{-1}$ frequency range. The reason for this difference in performance should be ascribed to the trajectory sampling distributions. In the case of the MC-SC-IVR simulations, the 14 trajectories were sampled in a harmonic fashion corresponding to the vibrational peaks of the first two frequencies ranges, while no one trajectory is such that its energy shell is located in the third range. Instead, for the TA-SC-IVR simulations the trajectories were sampled according to the Husimi distribution ${ }^{6,68}$ and so some of them have an energy within the third frequency range. In our previous calculations ${ }^{9}$ it was shown that the MC-SC-IVR can successfully detect peaks outside the energy shell covered by the few trajectories and this is confirmed by looking at the continuous (black) line in Fig. 5 in the frequency range of $3500-5500 \mathrm{~cm}^{-1}$.

Finally MC-SC-IVR simulations were performed on the system in Fig. 4 for a total of eight degrees of freedom. Our goal is to show how MC-SC-IVR can mimic the quantum dephasing and resonance originated by the long-range dipole interactions. To reach such a goal, one out of the four molecules was changed in isotopic composition from the standard ${ }^{12} \mathrm{C}^{16} \mathrm{O}$. If the four molecules are different, one should observe dephasing. Instead, if they are equal, the system should resonate as the one in Fig. 5. In Fig. 6 are reported the spectra for different isotopic compositions and even for noninteracting molecules. The last case, in which the dipole coupling has been switched off, corresponds to panel (a). For this case no splitting can be observed for either the external or internal stretch modes. In panel (b) the case where one molecule isotopic composition is set to ${ }^{14} \mathrm{C}^{18} \mathrm{O}$ is presented. In this case, dephasing can be observed by noting that both internal and external stretch frequencies are split. In panels (c) and (d) the isotopic composition is gradually changed to ${ }^{14} \mathrm{C}^{16} \mathrm{O}$ and ${ }^{12} \mathrm{C}^{18} \mathrm{O}$, respectively, and dephasing is gradually quenched. In panel (e) the fully resonating system is reported. In this case, no splitting is detected when only external stretch frequencies are excited, ${ }^{80}$ while splitting occurs in the second frequencies range, i.e., $1900-3500 \mathrm{~cm}^{-1}$ and higher ones, on the same fashion of the spectrum reported in Fig. 5.

In order to test such a long-range dipole interaction potential for an even more realistic system, the normal mode frequencies were calculated by Hessian diagonalizations for several adsorbed molecules. In Fig. 7 results are reported for 0.5 ML coverage consisting of a different number of molecules on different substrates. The choice of the substrate dimension, i.e., the number of copper atoms, was done so as to limit border effects. After several tests, we noticed that as a rule of thumb one can safely take a ratio of $\mathrm{CO}$ and surface $\mathrm{Cu}$ atoms number of about $\frac{1}{4}$, i.e., excluding that the edge presence modifies the internal stretch frequencies distribution of the half-monolayer. The main result of Fig. 7 is that 


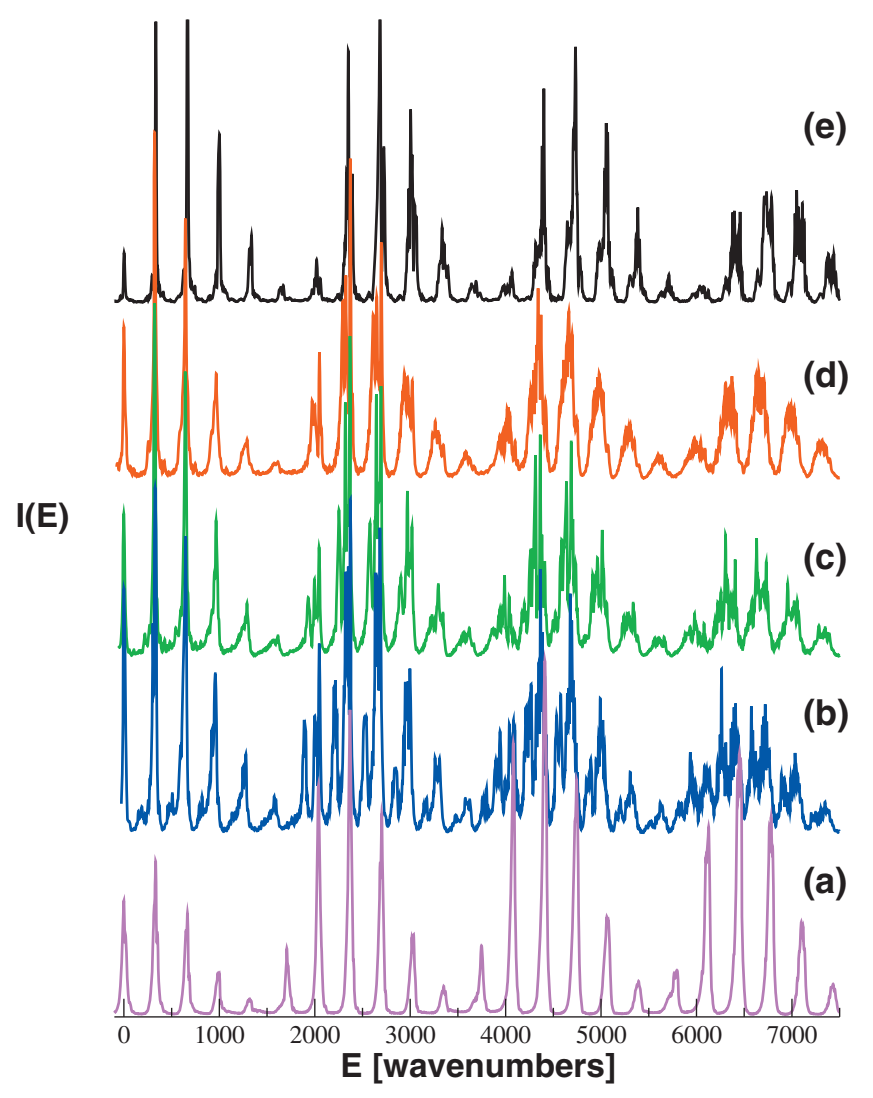

FIG. 6. Spectra for different isotopic compositions of a single CO molecule with respect to the other three in the system represented in Fig. 4: (a) four identical molecules without dipole coupling; (b) one molecule set to ${ }^{14} \mathrm{C}^{18} \mathrm{O}$; (c) one molecule set to ${ }^{14} \mathrm{C}^{16} \mathrm{O}$; (d) one molecule set to ${ }^{12} \mathrm{C}^{18} \mathrm{O}$; (e) resonating composition of four identical ${ }^{12} \mathrm{C}^{16} \mathrm{O}$ molecules dipole coupled.

frequencies are centered around $2068 \mathrm{~cm}^{-1}$, which is about $13 \mathrm{~cm}^{-1}$ less than the singleton frequency value. Instead, for two molecules the internal stretches were split such that one is at about $2041 \mathrm{~cm}^{-1}$ and the other at $2119 \mathrm{~cm}^{-1}$.

At this stage one can attempt a closer look at the experimental results. Pioneering experiments of $\mathrm{CO}$ on $\mathrm{Pt}(111)$ surfaces detected a single strong band shift from $2070 \mathrm{~cm}^{-1}$ toward lower frequency, as the surface coverage was decreased. When isotopic mixtures of ${ }^{12} \mathrm{CO}$ and ${ }^{13} \mathrm{CO}$ were adsorbed, then a second band was observed arising at $2000 \mathrm{~cm}^{-1} \cdot{ }^{11,14,15}$ The positions of the two bands depend on the mixture and intensity borrowing was observed. ${ }^{81}$ In our calculations the splitting slightly depends on the coverage since Fig. 5 shows a wider split than Fig. 6 for the internal modes. However, both values are comparable with the experimental frequency shift described above. No experiment could detect higher vibrational overtones and external overtones reported in Fig. 5 for the 14000 trajectory absorption cross-section, while the MC-SCIVR semiclassical spectra provide information about peak locations and not about peaks width and intensities. Finally, Fig. 7 can also be compared with experimental results. Considering that the singleton frequency was set at $2080 \mathrm{~cm}^{-1}$, then these results, that show lower frequency peaked distributions as the coverage is increased, are opposite in trend with respect to the experiments described above. ${ }^{11,14,15}$ This limitation resides not on the harmonic approximation but, most probably, on the po-
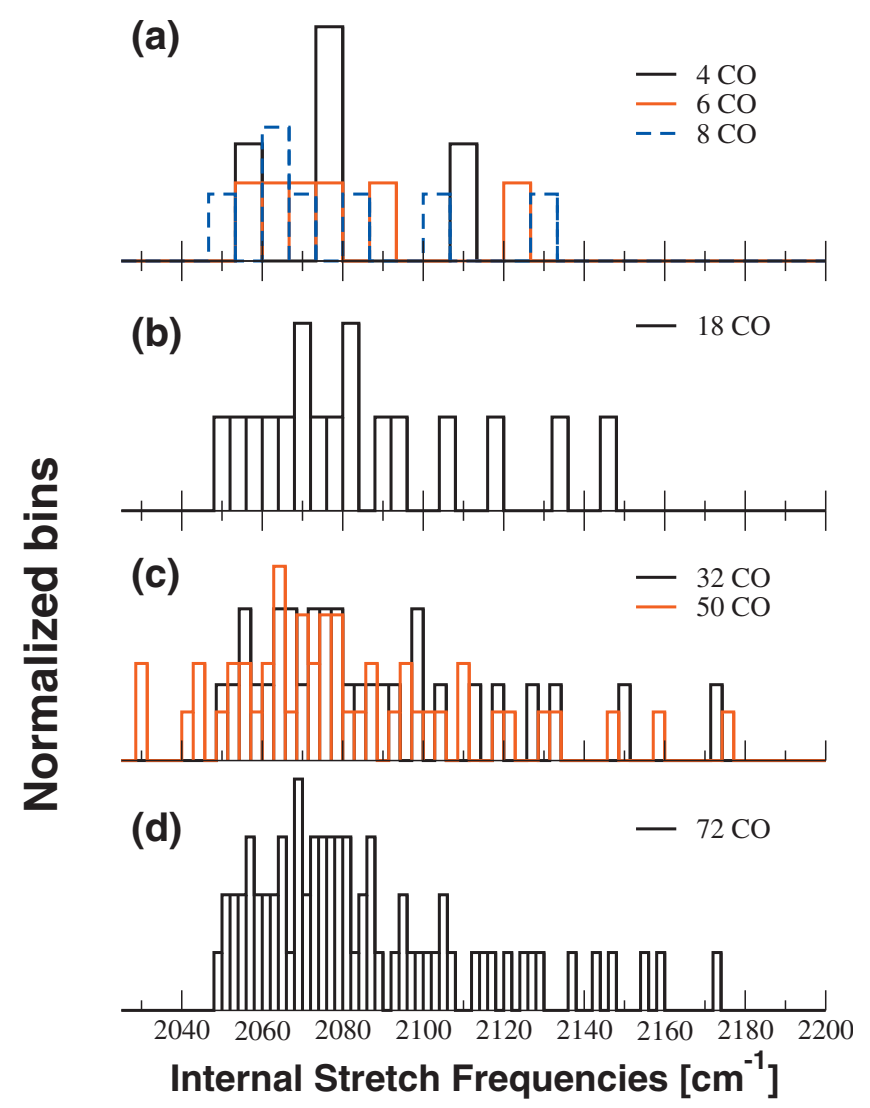

FIG. 7. Internal stretch harmonic frequencies normalized distributions for $\mathrm{CO}$ molecules adsorbed in a $c(2 \times 2)$ fashion. Labels indicate the number of $\mathrm{CO}$ molecules and (a) is for a substrate of $36 \mathrm{Cu}$ atoms; (b) for a substrate of $128 \mathrm{Cu}$ atoms; (c) for a substrate of $200 \mathrm{Cu}$ atoms; (d) for a substrate of $256 \mathrm{Cu}$ atoms.

tential energy surface itself. Such a difference in the frequency distribution is important if one has to consider the construction of a potential energy surface suitable for many $\mathrm{CO}$ molecule calculations. Nevertheless, when experimental isotopic mixtures are used as in Fig. 6, theoretical calculations predict the proper internal stretch peak shift. ${ }^{15}$

\section{CONCLUSIONS}

In this paper Tully's potential was revisited and enriched with long-range dipole interactions in order to properly describe adsorption monolayers. A preliminary classical analysis both for anharmonicity and for isotopic effects was pursued to assess the potential form. Subsequently quantum effects were included in the spectra calculations by using an implementation of the SC-IVR (MC-SCIVR) recently developed in our group. Such simulations showed that MC-SCIVR reasonably mimics the full SC-IVR results when these are available. It was found that on a system of four adsorbed $\mathrm{CO}$ molecules, the adsorbed layer is strongly resonating. Dephasing sets in when isotopic substitution is performed. Finally a more realistic arrangement was set up by using several $\mathrm{CO}$ molecules on larger $\mathrm{Cu}$ layers and harmonic analysis was performed. The statistical distributions of the internal stretching frequencies are peaked at $2068 \mathrm{~cm}^{-1}$ and not regularly distributed around this peak. This is probably due to the fact that the $\mathrm{CO}$ half-monolayer is limited and its 
border frequency induces such irregularity. Nevertheless, one should focus on the more peaked value for a comparison with experimental data. By taking panel (d) of Fig. 7 as the more realistic (harmonic) results, one can see that the actual frequency shift is about $\frac{1}{4}$ of that observed for a pair of molecules in our theoretical calculations and by experiments. However, in the experimental results the shifting depends not only on the substrate but also on the coverage. ${ }^{14,16}$ In conclusion, we observed that even if a dipole interaction can reproduce experimental frequency shifts for few adsorbed molecules, this is not necessarily true for a more complex and realistic adsorption setup.

\section{ACKNOWLEDGMENTS}

We warmly thank Dr. Vinod Krishna and Miss Stephanie Valleau for critically reading the manuscript. The University of Milan is thanked for funding (PUR grant) and CILEA (Consorzio Interuniversitario Lombardo per L'Elaborazione Automatica) for computational time allocation.

${ }^{1}$ X.-G. Wang and T. Carrington, Jr., J. Chem. Phys. 130, 094101 (2009).

${ }^{2}$ R. B. Lehoucq, S. K. Gray, D.-H. Zhang, and J. C. Light, Comput. Phys. Commun. 109, 15 (1998)

${ }^{3}$ J. Echave and D. C. Clary, Chem. Phys. Lett. 190, 225 (1992).

${ }^{4}$ J. Tennyson and J. R. Henderson, J. Chem. Phys. 91, 3815 (1989); B. T. Sutcliffe and J. Tennyson, Int. J. Quantum Chem. 39, 183 (1991); C. J. Tennyson and B. T. Sutcliffe, ibid. 42, 941 (1992).

${ }^{5}$ A. B. McCoy, Int. Rev. Phys. Chem. 25, 77 (2006)

${ }^{6}$ A. L. Kaledin and W. H. Miller, J. Chem. Phys. 118, 7174 (2003); M. Ceotto, Ph.D. dissertation, University of California, 2005; A. L. Kaledin and W. H. Miller, J. Chem. Phys. 119, 3078 (2003).

${ }^{7}$ M. Ceotto, S. Atahan, S. Shim, G. F. Tantardini, and A. Aspuru-Guzik, Phys. Chem. Chem. Phys. 11, 3861 (2009).

${ }^{8}$ J. M. Bowman, S. Carter, and X. Huang, Int. Rev. Phys. Chem. 22, 533 (2003); X. C. Huang, A. B. McCoy, J. M. Bowman, L. M. Johnson, C Savage, F. Dong, and D. J. Nesbitt, Science 311, 60 (2006).

${ }^{9}$ M. Ceotto, S. Atahan, G. F. Tantardini, and A. Aspuru-Guzik, J. Chem. Phys. 130, 234113 (2009).

${ }^{10}$ Y.-T. Wong and R. Hoffmann, J. Phys. Chem. 95, 859 (1991).

${ }^{11}$ R. M. Hammaker, S. A. Francis, and R. P. Eischens, Spectrochim. Acta 21, 1295 (1965)

${ }^{12}$ J. C. Tracy, J. Chem. Phys. 56, 2748 (1972)

${ }^{13}$ In gas phase two $\mathrm{CO}$ molecules best configuration would be T-like with $\mathrm{O}$ inserting $\mathrm{CO}$ bond; A. van der Pol, A. van der Avoird, and P. E. S. Wormer, J. Chem. Phys. 92, 7498 (1990).

${ }^{14}$ R. A. Shigeishi and D. A. King, Surf. Sci. 58, 379 (1976).

${ }^{15}$ A. Crossley and D. A. King, Surf. Sci. 68, 528 (1977).

${ }^{16}$ H. Pfnür, D. Menzel, F. M. Hoffmann, A. Ortega, and A. M. Bradshaw, Surf. Sci. 93, 431 (1980)

${ }^{17}$ B. J. N. Persson and R. Ryberg, Phys. Rev. B 24, 6954 (1981).

${ }^{18}$ B. N. J. Persson, Surf. Sci. 116, 585 (1982).

${ }^{19}$ B. N. J. Persson, J. Electron Spectrosc. Relat. Phenom. 29, 43 (1983).

${ }^{20}$ B. N. J. Persson, F. M. Hoffmann, and R. Ryberg, Phys. Rev. B 34, 2266 (1986).

${ }^{21}$ S. Andersson and B. N. J. Persson, Phys. Rev. Lett. 45, 1421 (1980).

${ }^{22}$ B. N. J. Persson and R. Ryberg, Phys. Rev. B 40, 10273 (1989).

${ }^{23}$ P. Jakob and B. N. J. Persson, Phys. Rev. B 56, 10644 (1997).

${ }^{24}$ M. Bonn, C. Hess, and M. Wolf, J. Chem. Phys. 115, 7725 (2001).

${ }^{25}$ R. Ryberg, Phys. Rev. B 32, 2671 (1985).

${ }^{26}$ G. Blyholder, J. Phys. Chem. 68, 2772 (1964).

${ }^{27}$ A. Föhlisch, M. Nyberg, P. Bennich, L. Triguero, J. Hasselström, O. Karis, L. G. M. Pettersson, and A. Nilsson, J. Chem. Phys. 112, 1946 (2000).

${ }^{28} \mathrm{CO}$ molecule dissociates on early transition metals.

${ }^{29}$ J. T. Kindt, J. C. Tully, M. Head-Gordon, and M. A. Gomez, J. Chem. Phys. 109, 3629 (1998).

${ }^{30}$ F. Dzegilenko, J. M. Bowman, and S. Carter, J. Chem. Phys. 109, 7506 (1998).
${ }^{31}$ S. C. Park, W. K. Park, and J. M. Bowman, Surf. Sci. 427-428, 343 (1999).

${ }^{32}$ S. Picaud, P. N. M. Hoang, C. Girardet, A. Meredith, and A. J. Stone, Surf. Sci. 294, 149 (1993).

${ }^{33}$ F. Favot, A. Del Corso, and A. Baldereschi, J. Chem. Phys. 114, 483 (2001).

${ }^{34}$ R. Marquardt, F. Cuvelier, R. A. Olsen, E. J. Baerends, J. C. Tremblay, and P. Saalfrank, J. Chem. Phys. 132, 074108 (2010).

${ }^{35}$ P. Fouquet, R. A. Olsen, and E. J. Baerends, J. Chem. Phys. 119, 509 (2003).

${ }^{36}$ A. Stroppa, K. Termentzidis, J. Paier, G. Kresse, and J. Hafner, Phys. Rev. B 76, 195440 (2007)

${ }^{37}$ J. C. Tully, M. Gomez, and M. Head-Gordon, J. Vac. Sci. Technol. A 11, 1914 (1993)

${ }^{38}$ V. Krishna and J. C. Tully, J. Chem. Phys. 125, 054706 (2006).

${ }^{39}$ S. Andersson, Solid State Commun. 21, 75 (1977).

${ }^{40}$ C. L. Allyn, T. Gustafsson, and E. W. Plummer, Chem. Phys. Lett. 47, 127 (1977).

${ }^{41}$ S. Andersson and J. B. Pendry, Surf. Sci. 71, 75 (1978).

${ }^{42}$ J. K. Nørskov, H. Hjelmberg, and B. I. Lundqvist, Solid State Commun. 28, 899 (1978).

${ }^{43}$ M. Passler, A. Ignatiev, F. Jona, D. W. Jepsen, and P. M. Marcus, Phys. Rev. Lett. 43, 360 (1979).

${ }^{44}$ C. F. McConville, D. P. Woodruff, K. C. Prince, G. Paolucci, V. Chab, M. Surman, and A. M. Bradshaw, Surf. Sci. 166, 221 (1986).

${ }^{45}$ C. J. Hirschmugl, G. P. Williams, F. M. Hoffmann, and Y. J. Chabal, J. Electron Spectrosc. Relat. Phenom. 54-55, 109 (1990).

${ }^{46}$ C. J. Hirschmugl, G. P. Williams, F. M. Hoffmann, and Y. J. Chabal, Phys. Rev. Lett. 65, 480 (1990).

${ }^{47}$ T. A. Germer, J. C. Stephensoe, E. J. Heilweil, and R. R. Cavanagh, Phys. Rev. Lett. 71, 3327 (1993)

${ }^{48}$ T. A. Germer, J. C. Stephenson, E. J. Heilweil, and R. R. Cavanagh, J. Chem. Phys. 101, 1704 (1994).

${ }^{49}$ C. J. Hirschmugl, Y. J. Chabal, F. M. Hoffmann, and G. P. Williams, J. Vac. Sci. Technol. A 12, 2229 (1994).

${ }^{50}$ R. Arafune, K. Hayashi, S. Ueda, Y. Uehara, and S. Ushioda, Surf. Sci. 600, 3536 (2006).

${ }^{51}$ S. Andersson and J. B. Pendry, Phys. Rev. Lett. 43, 363 (1979).

${ }^{52}$ See http://www.nist.gov/index.html.

${ }^{53}$ D. R. Lide, Handbook of Chemistry and Physics, 88th ed. (CRC, Boca Raton, FL and London, 2007).

${ }^{54}$ K. Hermann, P. S. Bagus, and C. W. Bauschlicher, Jr., Phys. Rev. B 30, 7313 (1984).

${ }^{55}$ J. C. Tremblay, S. Beyvers, and P. Saalfrank, J. Chem. Phys. 128, 194709 (2008).

${ }^{56}$ C. W. Muhlhausen, L. R. Williams, and J. C. Tully, J. Chem. Phys. 83, 2594 (1985)

${ }^{57}$ S. C. Park, J. M. Bowman, and D. A. Jelski, J. Chem. Phys. 104, 2457 (1996).

${ }^{58}$ S. Carter, S. J. Culik, and J. M. Bowman, J. Chem. Phys. 107, 10458 (1997).

${ }^{59}$ A. Bahel and Z. Bacic, J. Chem. Phys. 111, 11164 (1999).

${ }^{60}$ C. Cattarius and H.-D. Meyer, J. Chem. Phys. 121, 9283 (2004).

${ }^{61}$ S. Beyvers, Y. Ohsuki, and P. Saalfrank, J. Chem. Phys. 124, 234706 (2006).

${ }^{62}$ C. M. Truong, J. A. Rodriguez, and D. W. Goodman, Surf. Sci. Lett. 271, L385 (1992).

${ }^{63}$ S. P. Lewis and A. M. Rappe, Phys. Rev. Lett. 77, 5241 (1996).

${ }^{64}$ G. D. Mahan and A. A. Lucas, J. Chem. Phys. 68, 1344 (1978).

${ }^{65}$ A. R. Walton and D. E. Manolopoulos, Mol. Phys. 87, 961 (1996); Chem. Phys. Lett. 244, 448 (1995); M. L. Brewer, J. S. Hulme, and D. E. Manolopoulos, J. Chem. Phys. 106, 4832 (1997).

${ }^{66}$ W. H. Miller, Adv. Chem. Phys. 25, 69 (1974); Faraday Discuss. 110, 1 (1998)

${ }^{67}$ W. H. Miller, J. Chem. Phys. 53, 3578 (1970); 53, 1949 (1970); J. Phys. Chem. A 105, 2942 (2001); M. Thoss and H. Wang, Annu. Rev. Phys. Chem. 55, 299 (2004); K. G. Kay, ibid. 56, 255 (2005).

${ }^{68}$ H. Wang, X. Sun, and W. H. Miller, J. Chem. Phys. 108, 9726 (1988); X. Sun and W. H. Miller, ibid. 110, 6635 (1999); M. Thoss, H. Wang, and W. H. Miller, ibid. 114, 9220 (2001); T. Yamamoto, H. Wang, and W. H. Miller, ibid. 116, 7335 (2002); T. Yamamoto and W. H. Miller, ibid. 118, 2135 (2003)

${ }^{69}$ M. Topaler and N. Makri, J. Chem. Phys. 101, 7500 (1994); K. Thompson and N. Makri, ibid. 110, 1343 (1999); N. Makri, Annu. Rev. Phys. 
Chem. 50, 167 (1999); N. J. Wright and N. Makri, J. Chem. Phys. 119, 1634 (2003).

${ }^{70}$ J. Ankerhold, M. Saltzer, and E. Pollak, J. Chem. Phys. 116, 5925 (2002); S. Zhang and E. Pollak, Phys. Rev. Lett. 91, 190201 (2003); J. Chem. Phys. 121, 3384 (2004)

${ }^{71}$ S. Bonella, D. Montemayor, and D. F. Coker, Proc. Natl. Acad. Sci. U.S.A. 102, 6715 (2005); S. Bonella and D. F. Coker, J. Chem. Phys. 118, 4370 (2003)

${ }^{72}$ Y. Wu, M. Herman, and V. S. Batista, J. Chem. Phys. 122, 114114 (2005); Y. Wu and V. S. Batista, ibid. 118, 6720 (2003)

${ }^{73}$ F. Grossmann, Comments At. Mol. Phys. 34, 243 (1999).

${ }^{74}$ E. J. Heller, J. Chem. Phys. 62, 1544 (1975); 75, 2923 (1981).
${ }^{75}$ M. F. Herman and E. Kluk, Chem. Phys. 91, 27 (1984); K. G. Kay, J. Chem. Phys. 100, 4377 (1994); 100, 4432 (1994).

${ }^{76}$ E. J. Heller, Acc. Chem. Res. 14, 368 (1981); 39, 127 (2006).

${ }^{77}$ The IR absorption is proportional to $E \times \mathrm{I}(E)$, while the IR emission spectrum is proportional to $E^{3} \times \mathrm{I}(E)$.

${ }^{78}$ Y. Elran and K. G. Kay, J. Chem. Phys. 110, 3653 (1999); 110, 8912 (1999).

${ }^{79}$ A. L. Kaledin, X. Huang, and J. M. Bowman, Chem. Phys. Lett. 384, 80 (2004).

${ }^{80} \mathrm{~A}$ mild splitting gradually sets in for external mode excitations and it is evident only for the sixth overtone.

${ }^{81}$ E. Borguet and H.-L. Dai, Chem. Phys. Lett. 194, 57 (1992). 